\title{
Biosynthesis of Silver Nanoparticles by Green Chemistry from Ectoine-compatible Solute
}

\author{
Sulochana $\mathrm{MB}^{*}$ and Parameshwar AB \\ Department of Research and Studies in Biotechnology, Gulbarga University, Kalaburgi-585106, Karnataka, India
}

${ }^{*}$ Corresponding author: Sulochana MB, Department of Research and Studies in Biotechnology, Jnana Ganga, Sedam Road, Gulbarga University, Kalaburgi-585106, Karnataka, India; Email: mbsulochana@rediffmail.com

Received: February 03, 2022; Accepted: February 10, 2022; Published: February 17, 2022

\begin{abstract}
The unique ultrafast easy and consistent bio synthesis process was established for the synthesis of nanoparticles using haloalkaliphilic bacteria. The strain used was Halomonas organivorans, which was characterized by $16 \mathrm{~S}$ rRNA sequencing having the NCBI Accession No. JQ906721. This strain accumulates the compatible solutes - ectoine in response to high salinity. In the present study ectoine based silver nanoparticles was obtained within seconds which showed rapid synthesis of nanoparticles using sunlight. Synthesis of the silver $(\mathrm{Ag})$ nanoparticles is aimed at the innovative probable artistic applications in the field of Bio-nanotechnology. Sunlight was converged using convex lens into the sample containing $\mathrm{AgNO}_{3^{\prime}}$ compatible solute - ectoine. Rapid change in colour was observed in 3-5 seconds which designated the formation of silver nanoparticles and was further characterized by UV-visible spectroscopy, FTIR and AFM.
\end{abstract}

Keywords: Ectoine, Halomonas organivoarans, UV-Vis. and AFM

\section{Introduction}

Development of consistent technology to produce nanoparticles is an important aspect of nanotechnology. Biological synthesis provides a wide range of environmentally suitable procedure, lowcost production with minutest time required. Biosynthesis of silverbased nanoparticles fascinated the attention of the Scientists for the ultrafast green synthesis. Due to the unique physicochemical properties and their potential applications in biotechnology, medical biotechnology, material science, chemistry and physics have led to the revolution of synthesizing novel generation miniscule particles with noble properties. The thirst among the researchers for the new approaches for the synthesis of silver nanoparticles was observed for the antimicrobial activity. Several biological methods employed for the synthesis are eco-friendly in nature and are nontoxic to environment, whereas chemical methods are dangerous to environment [1]. There are substantial reports suggesting the use of biological material for the synthesis of nanoparticles by bacteria, fungi, plant extract and yeast. Recently Scientists have used microorganisms and their intracellular and extracellular preparations for the synthesis of silver nanoparticles and whole cells were also known to reduce $\mathrm{Ag}+$ ions to produce silver nanoparticles.

In our present investigation, marine isolate Halomonas organivornas accumulates higher quantity of compatible solutes in response to counter balance the extracellular $\mathrm{NaCl}$ concentration. Compatible solute based ultrafast green synthesis of silver nanoparticles was carried out by convergence of the irradiated sunlight into the solution resulting in the formation of silver nanoparticles within (3-5) seconds.

\section{Materials and Methods}

\section{Bacterial Culture}

The strain Halomonas organivorans was isolated in our laboratory and deposited in (NCBI Accession No. JQ906721) cultivated in Halophilic media containing $10 \mathrm{~g}$ Peptone, $10 \mathrm{~g}$ Yeast extract, $20 \mathrm{~g}$ $\mathrm{MgSO}_{4} 2 \mathrm{~g} \mathrm{KCl}, 3 \mathrm{~g}$ tri-sodium citrate, incubated at $37^{\circ} \mathrm{C}$ for $24 \mathrm{~h}$. $H$. organivorans was then inoculated into $100 \mathrm{ml}$ Halophilic broth and incubated at $37^{\circ} \mathrm{C}$ in a shaking incubator. The bacterial biomass obtained was centrifuged at $10,000 \mathrm{rpm}$ for $10 \mathrm{~min}$ at $4^{\circ} \mathrm{C}$ and was used for the extraction of the osmolytes.

\section{Purification of the Compatible Solute-ectoine}

$50 \mathrm{ml}$ of the bacterial culture was centrifuged at 10,000 rpm for 10 min at $4^{\circ} \mathrm{C}$ cell pellet obtained was suspended in the double distilled sterile water for $20 \mathrm{~min}$. The extract was separated by repeating the centrifugation step and the supernatant was collected. The extract obtained was filtered through $0.2 \mu$ filter and finally a colourless extract was obtained and scanned by UV-VIS for the detection of synthesis of nanoparticles (UV region $210-230 \mathrm{~nm}$ ) and stored at $4^{\circ} \mathrm{C}$ until further use.

\section{Biosynthesis of Silver Nanoparticles}

In a typical biosynthesis of silver nanoparticles, $1 \mathrm{ml}$ of compatible solute-ectoine was mixed with $20 \mathrm{ml}$ aqueous solution of $1 \mathrm{mM}$ silver nitrate (AgNO3). The sunlight was converged using the convex lens into the sample mixture then within 3-5 seconds change in colour of the solution to brown was observed, thus, this indicated the formation of silver nanoparticles. 


\section{Optimization of Reaction Time}

Silver nanoparticle synthesis was assessed at different reaction time of 2, 3, 4, 5 and 60 Sec using UV-Vis spectroscopy which showed the colour changes of silver nanoparticles in different reaction. Sunlight irradiation time was accurately monitored, increase in the time of exposure led to the formation of particle agglutination and thus rapid change in colour was measured indicating the formation of silver nanoparticles.

\section{Characterization of Nanoparticles UV-VIS Visible Analysis}

The reduction of silver ions was monitored by visual observation, actual reduction and formation of nanoparticles were examined by the UV-VIS spectroscopy scan from $200 \mathrm{~nm}$ to $800 \mathrm{~nm}$.

\section{Fourier Transform Infrared Spectroscopy (FTIR) Analysis}

FTIR is highly diverse molecular spectroscopy technique employed for the analysis of biological and chemical properties. AgNPs are reduced with different biomolecules in the reaction mixture, hence understanding the precise functional group of biomolecules produced and FTIR spectra shed more information on nature of the AgNPs synthesized and were well documented in various studies. FTIR System used in this study was Shimadzu FTIR-8201 PC instrument and it was run in the diffuse reflectance mode at a resolution of $4 \mathrm{~cm}^{-1}$ in KBR pellets.

\section{Atomic Force Microscope (AFM) Studies}

The samples were diluted to 10 times with distilled water and then dropped onto the glass slides, followed by vacuum drying at $30^{\circ} \mathrm{C}$ for $24 \mathrm{~h}$. The measurements of the height of nanoparticles were observed by AFM image analysis software. The surface properties of the biosynthesized nanoparticles were visualized by an atomic force microscope, under the normal atmospheric conditions. Explorer atomic force microscope was in tapping mode, using high-resonantfrequency for the analysis, the study was carried out at central instrumentation center, Karnataka University, Dharwad. AFM imaging in UHV and in ambient air, the dynamic mode (DM-AFM), sometimes also termed the "tapping mode" is the method of choice for imaging surfaces.

\section{Scanning Electron Microscope (SEM) Studies}

The bacterial cells of Halomonas organivorans were obtained by centrifuging at 5,000 rev/min and the cells were washed twice with potassium phosphate buffer (50 mM, pH 7.0). Bacterial cells were then fixed with immersion in $2.5 \%$ glutaraldehyde in potassium phosphate buffer $(50 \mathrm{mM}, \mathrm{pH} 7)$ overnight at $4^{\circ} \mathrm{C}$. The specimens were washed twice with buffer and dehydrated with an ethanol series (v/v) ranging from 30, 40, 50, 60, 70, 80, 90 and $100 \%$ and stored in $100 \%$ ethanol. For SEM, the specimens were dried to critical point, coated with gold and examined with an S-200C scanning electron microscope.

\section{Transmission Electron Microscopy (TEM) Studies}

Transmission electron micrographs reveals the morphology of the nanoparticles under examination, substantial morphology of the silver nanoparticles synthesized were spherical in shape, appeared in bulk and this was carried out at SAIF, Cochin. TEM studies were performed using an electron microscope operating at an accelerating voltage of $90 \mathrm{kV}$. The dimensions of the silver nanoparticles were carried out by TEM by adding a drop of the solution containing the particles and was placed on a copper grid covered with amorphous carbon. Allow the film to stand for $2 \mathrm{~min}$ and the extra solution was removed by means of blotting paper and the grid was allowed to drying before using in the microscope. The nanoparticle films were also formed on carbon coated copper grids $(40 \mu \mathrm{m} \times 40 \mu \mathrm{m}$ mesh size $)$ and transmission electron microscopy (TEM) images of the films were scanned on a JEOL 1200 EX instrument operated at an accelerated voltage of $120 \mathrm{kV}$.

\section{Results and Discussion}

In the present study synthesis of AgNPs from compatible solute - Ectoine was produced by Halomonas organivorans (No. JQ906721) and the phylogenetic tree as shown in Figure 1. this study is reported for the first-time using convergence of the irradiated sunlight as per our knowledge as shown in Figure 2. We are contributing a simple ultrafast green biosynthesis of AgNPs using converged photon irradiation. AgNPs have appeared as a promising candidate in the field of medical because of their physically illustrious size and shape, nanoparticles exhibit different properties when compared with the bulk material.
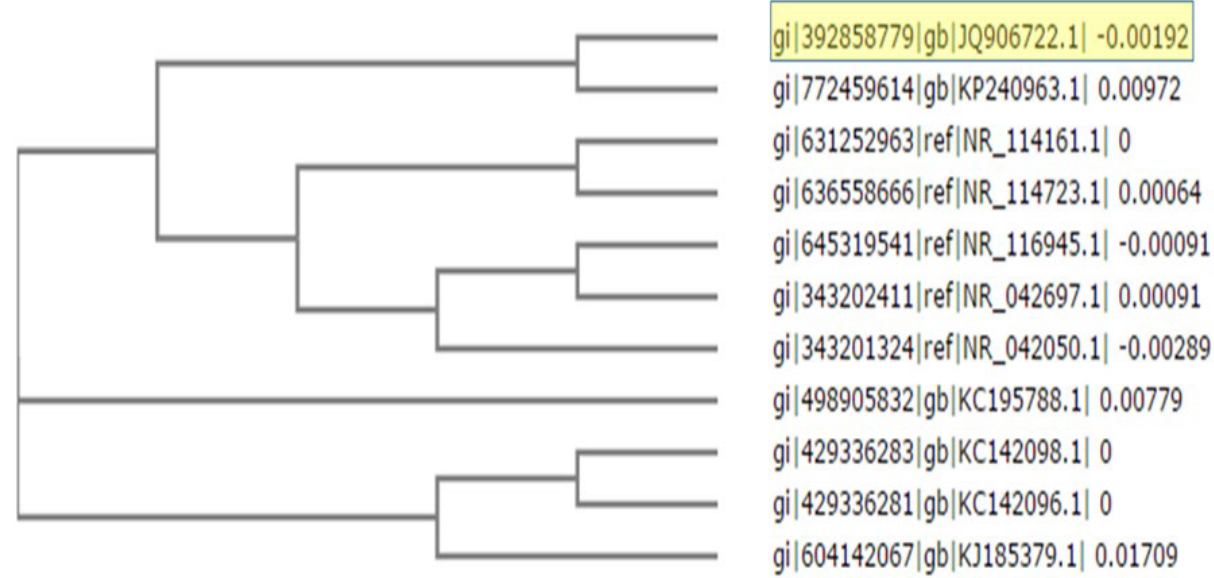

Figure 1: Phylogenetic tree of Halomonas organivorans (No. JQ906721). 

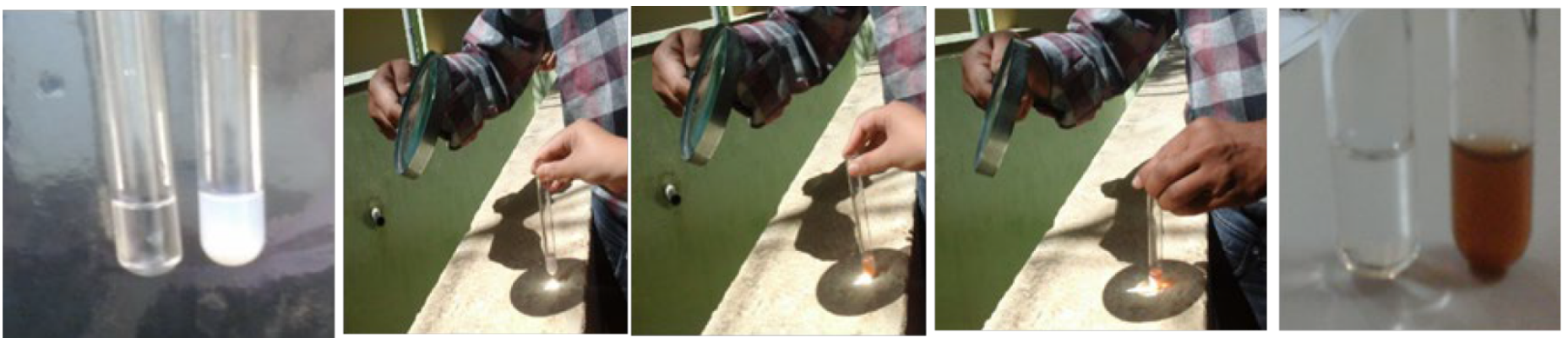

Figure 2: Synthesis of silver nanoparticles with photon irradiation.

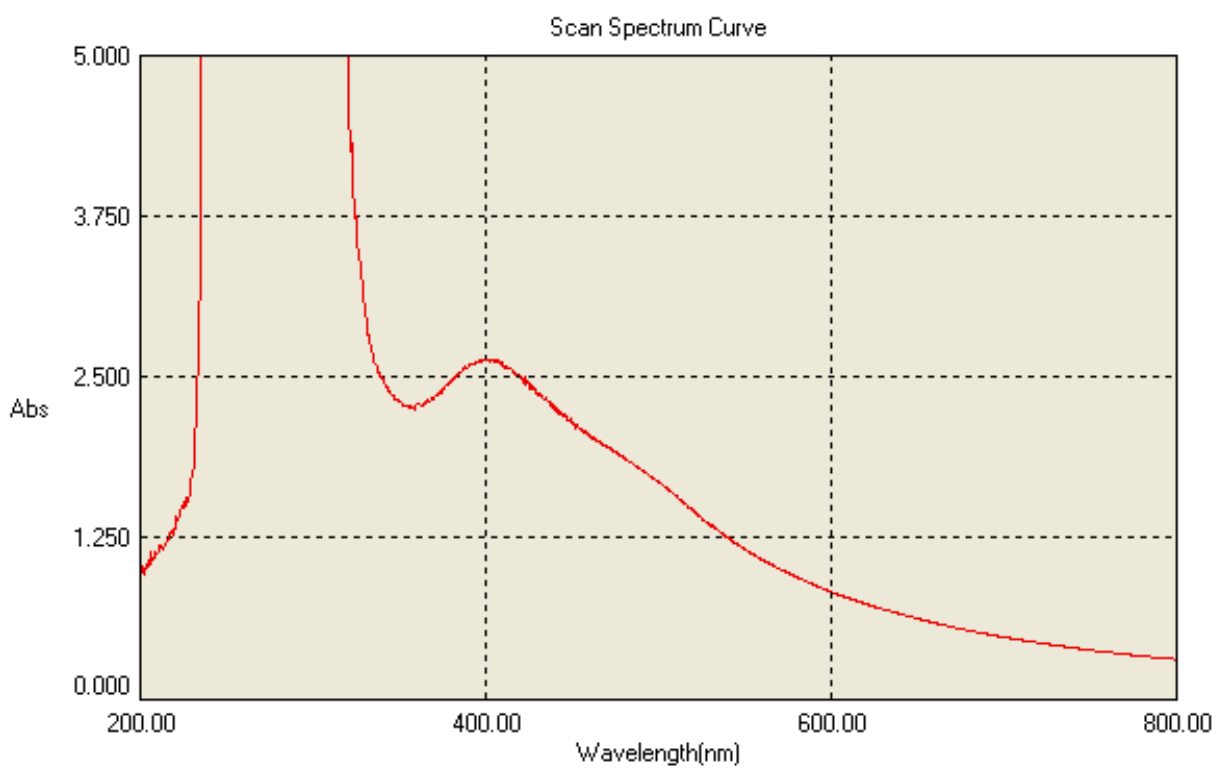

Figure 3: UV Spectral Characterization of nanoparticles.

Synthesis of the nanoparticles was carried out by chemical, biological and radiation methods, but there is always a scope for the development of easy, swift, safe and green synthesis of Nanoparticles. Hence, our existing study proves to be a noteworthy ultrafast, easy economic step in the of biological synthesis of nanoparticles.

\section{UV-VIS Visible Analysis}

This technique is based on the Surface Plasmon Resonance phenomenon (SPR), the change in colour of the nanoparticles has its derivation in collective electron excitation when related to external excitation which arises when external electromagnetic wave focuses on the particle. This creates an oscillation and each wavelength produces different oscillations in the electron cloud, which may be resonant or non-resonant. The specific wavelength of the electromagnetic radiation converted into thermal energy is the SPR peak obtained.

In the current work extracellular biosynthesis of AgNPs using the compatible solute ectoine extract and $20 \mathrm{ml}$ of $1 \mathrm{mM} \mathrm{AgNO} 3$ were used. Colour changed from white to dark-brown within 3-5 sec after exposure to the condensed sunlight using convex lens. The UV-Vis spectrophotometer analysis showed the absorbance peak at round $400 \mathrm{~nm}$ as shown in Figure 3 which was specific for the synthesis of AgNPs. These findings are similar to the report of [2], in which the
Capsicum annuum $L$. extract reacted with aqueous silver ions, the reaction mixture containing AgNPs showed the absorption peak at about $410 \mathrm{~nm}$ due to the excitation of longitudinal plasmon resonance vibration.

The maximum absorbance band at $412 \mathrm{~nm}$ [3] showed the surface plasmon resonance band for silver colloid thus the peak confirms the formation of spherical structures of the silver nanoparticles [4]. The intensity of the absorbed peak for silver nanoparticle exhibits the peculiar surface plasmon resonance peak between 400 to $430 \mathrm{~nm}$ was well known phenomenon for AgNP characterization in various studies. Most of the studies use UV Visible to identify the presence and production of Nanoparticles as well identify the size and shape of the NP $[5,6]$.

\section{Fourier Transform Infrared Spectroscopy (FTIR)}

The biomolecule responsible for the reduction and formation of silver nanoparticles was identified by evaluating and interpreting the FTIR data analysis. The IR bands observed at $3,427 \mathrm{~cm}^{-1}$ indicated the O-H stretch of carboxylic acid, or phenols, band at $2924 \mathrm{~cm}^{-1}$ corresponding to $\mathrm{C}-\mathrm{H}$ stretch and the bands at $1,626 \mathrm{~cm}^{-1}$ corresponding to primary and secondary amides $[7,8]$. And $1384 \mathrm{~cm}^{-1}$ indicated the presence of methyl group, the bands in the fingerprint region at 1102 , 
$1023 \mathrm{~cm}^{-1}$ is as shown in Figure 4 which represented the involvement of $\mathrm{C}-\mathrm{N}$ aliphatic amine assessment from the bands indicated the presence of peptide bound AgNPs. Hence the conceivable reduction with the ectoine molecules in the compatible solute occurred.

\section{Atomic Force Microscopy (AFM) Studies}

Atomic force microscopy (AFM) documents the imaging of the surface of samples on a nanometer scale in ultrahigh vacuum (UHV), ambient air, and liquids. The measurement of very small structures by AFM in air implies a relatively constant environment in terms of temperature and humidity. The slightest air drift or temperature shift during the measurement can cause a drift in the image. Therefore, an isolating box was built around the microscope which maintains a constant humidity inside and keep the temperature fluctuations to minimum. In addition, the box containing the microscope was put on top of an active damping table to prevent vibration. Humidity was reduced by placing silica gel inside the box; humidity and temperature were monitored by a Lab-view programme. Imaging was done in the non-contact dynamic mode at ambient conditions with humidity of $30-40 \%$ and all the images have been processed for better quality.

Atomic force microscopy has been used to study the silver nanoparticles morphology and surface topology as shown in Figure 5. AgNP's are spherical in shape and exhibit smooth surface which was observed by atomic force microscope under tapping mode. The silver nanoparticles prepared from the compatible solute solution showed smooth topology as recorded by [9]. AFM Surface morphology of the formulated nanoparticles studied under AFM are displaying spherical shape of nanoparticles with smooth surface, without any pinholes or cracks.

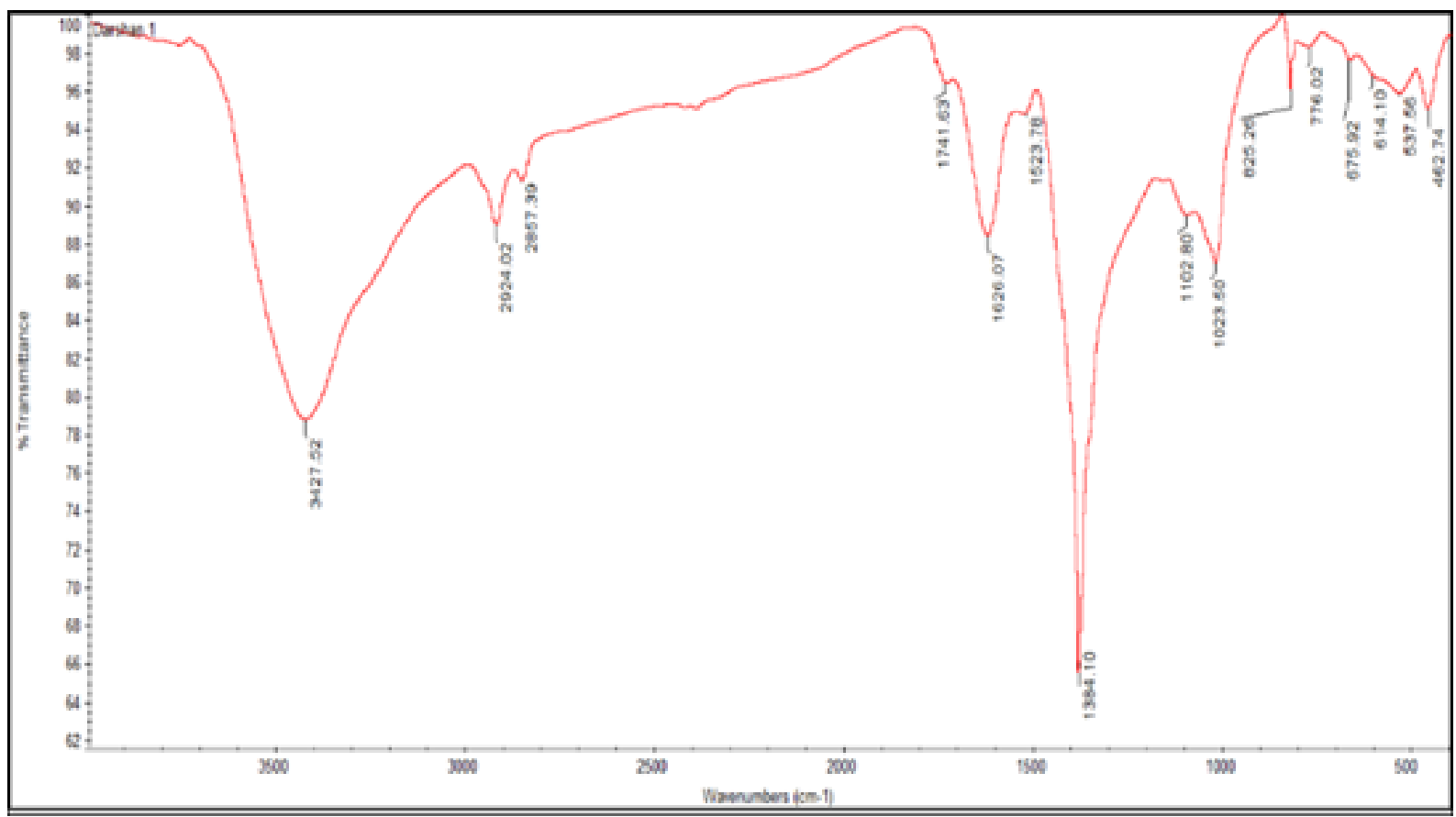

Figure 4: FTIR analysis of silver nanoparticles.
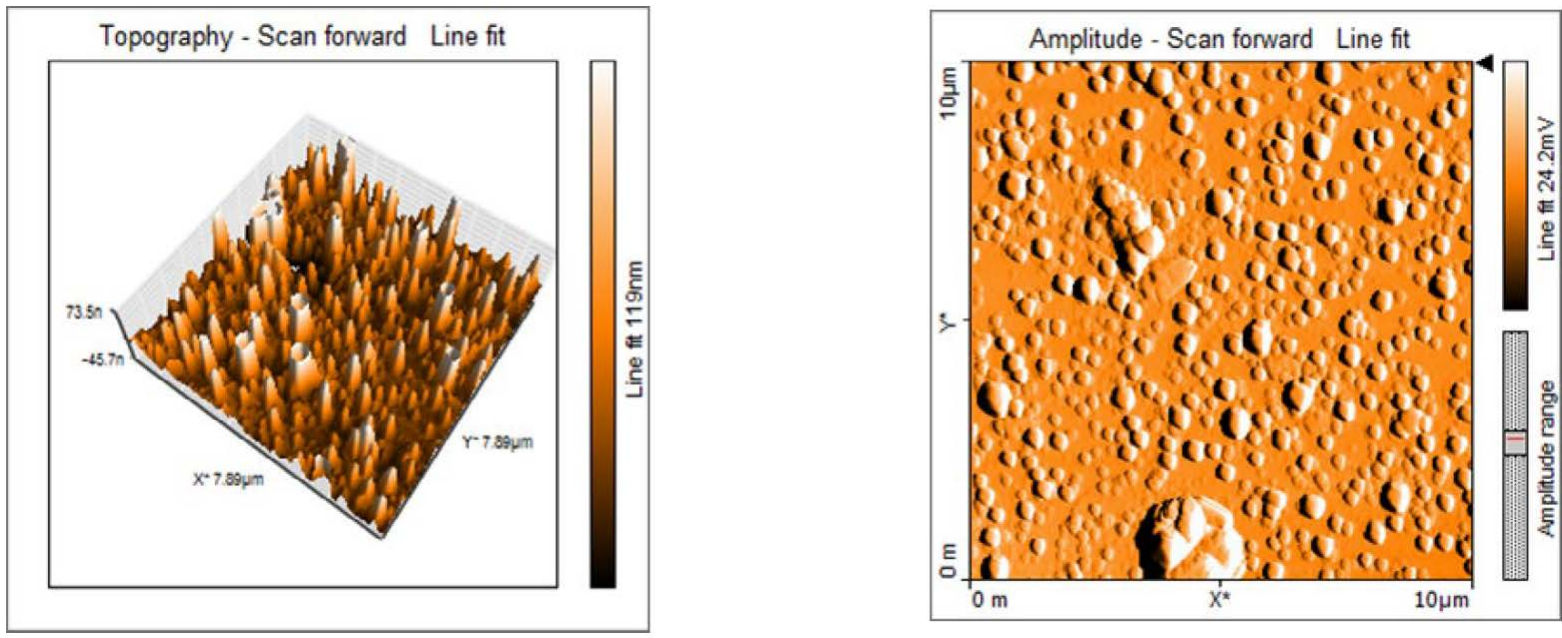

Figure 5: The topology of the Ectoine silver nanoparticles by AFM. 


\section{Scanning Electron Microscopy (SEM) studies}

The interpretation of scanning electron microscopy shows the size of the particles and they were nanosized as spherical in shape ranging from 1.63 to $1.85 \mu \mathrm{m}$ as shown in Figure 6. Predominantly most of the structures are spherical and the silver nanoparticles synthesized from the reaction of silver ions and compatible solutes were stable even after 1-2 months of storage. [10] the size of the nanoparticle synthesised were closely relevant with extracellular silver nanoparticles from Azadirachta indica (Plant) 50-100 nm. Extracellular nanoparticles from Colletotrichum sp. with $20-40 \mathrm{~nm}$ was reported by [11] and extracellular silver nanoparticles of Nitrate reductases from Fusarium oxysporum, was 10-35 $\mathrm{nm}$.

\section{Transmission Electron Microscopy}

Transmission Electron micrographs reveals the morphology of the nanoparticles which showed the shape and size of Nanoparticles of 30-
$55 \mathrm{~nm}$ with spherical shape and appeared in bulk as shown in Figure 7. This was similar to the result observed by Bacillus lichiniformis mediated silver nanoparticles reported by [12]. The Synthesis of nanoparticles outside the cell extracellularly has many applications and the microbial synthesis of the metal nanoparticles depends upon the localization of the reductive components of the cell. When the cell wall reductive enzymes or soluble secreted enzymes are involved in the reductive process of metal ions then it is obvious to find the metal nanoparticles. It is one of the simple and eco-friendly method when compared to the chemical and physical method as it is cost effective and there are no side effects.

\section{Conclusion}

- The present investigation demonstrates the rapid biosynthesis of silver nanoparticles from the compatible solutes ectoine using converged photon irradiation, an eco-friendly, green and ultrafast protocol for the biosynthesis of AgNP's.

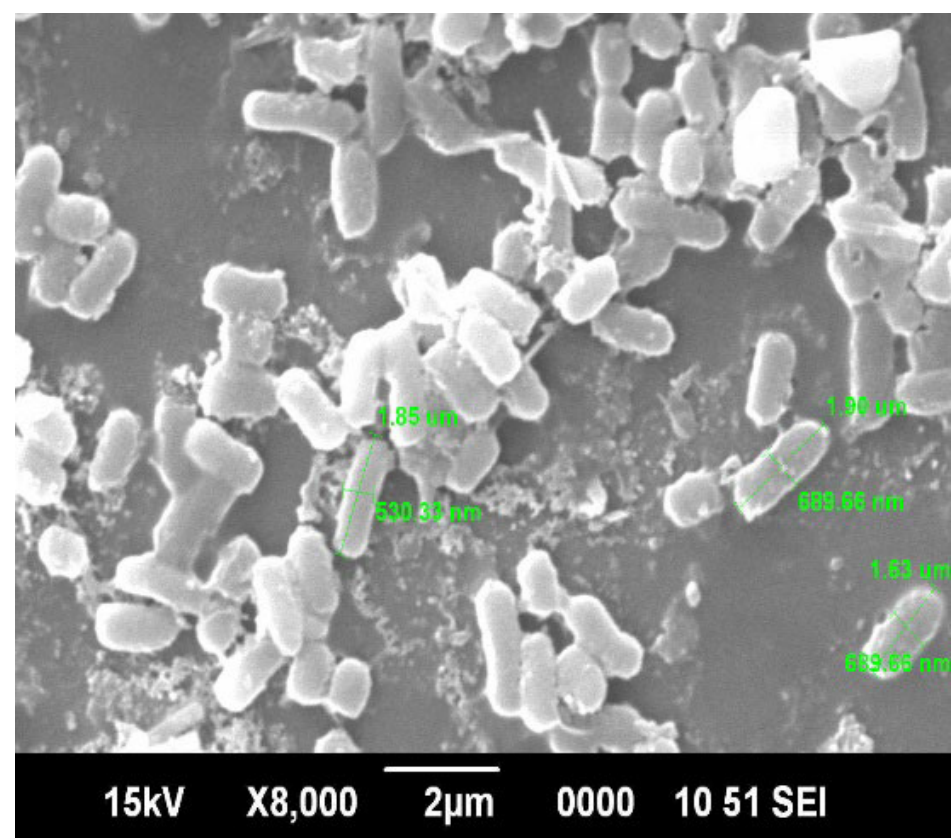

Figure 6: Scanning Electron Microscopy of silver nanoparticles.

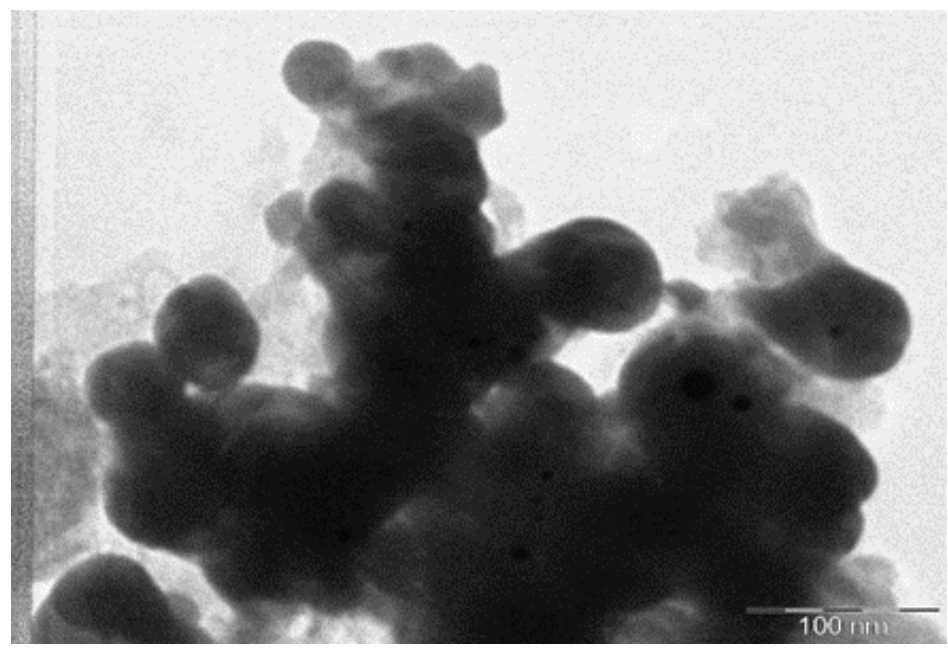

Figure 7: TEM of AgNPs biosynthesis from compatible solute. 
- Halomonas organivorans (No. JQ906721) stores ectoine intracellularly to encounter the high concentration of $\mathrm{NaCl}$ extracellularly. The reduction of silver nitrate to AgNPs is facilitated by the presence of the ectoine, a pyrimidine carboxylic acid present in the compatible solution.

- The Ectoine molecule present in the reaction mixture, in presence of high photon energy was reduced to ectoinebonded Silver Nanoparticles and was detected at the UV absorbance peak at $400 \mathrm{~nm}$. The intensity of the absorbed peak for silver nanoparticle exhibits the peculiar surface plasmon resonance peak between 400 to $430 \mathrm{~nm}$.

- This is the first report for the biosynthesis of AgNPs using converged photon irradiation using the biological method in just few seconds (3-5). There are no reports about the use of converged light for the synthesis of AgNPs, from the available literature, comparing to the current method of the synthesis, this method is very precise, swift and inexpensive.

- The O-H stretch of carboxylic acid, or phenols bands in the fingerprint region at $1102,1023 \mathrm{~cm}^{-1}$ represented the involvement of $\mathrm{C}-\mathrm{N}$ aliphatic amine assessment, from the bands indicated the presence of peptide bound AgNPs which were confirmed by (FTIR).

- AgNP's are spherical in shape and exhibit smooth surface which was observed by atomic force microscope under tapping mode. AFM Surface morphology of the formulated nanoparticles studied under AFM are displaying spherical shape of nanoparticles with smooth surface, without any pinholes or cracks.

- The interpretation of scanning electron microscopy (SEM) shows the size of the particles and they were nanosized as spherical in shape ranging from 1.63 to $1.85 \mu \mathrm{m}$. Predominantly most of the structures are spherical and the silver nanoparticles synthesized from the reaction of silver ions and compatible solutes were stable even after 1-2 months of storage.

Transmission Electron micrographs reveals the morphology of the nanoparticles which showed the shape and size of Nanoparticles of 30-55 nm with spherical shape and appeared in bulk. The increase in polydispersity and broad size distribution with increase in metal ion concentration was evident from TEM image. It consisted of almost uniformly sized spherical nanoparticles of average size of $15 \mathrm{~nm}$ with diameter ranging from 7 to $25 \mathrm{~nm}$.

\section{References}

1. Dubey SP, Lahtinen M, Sillanpaa E (2010b). Tansy fruit mediated greener synthesis of silver and gold nanoparticles. Process Biochem 45: 1065-1071.

2. Shikuo Li, Yuhua Shen, Anjian Xie, Xuerong Yu, Lingguang Qiu, et al. (2007) Green synthesis of silver nanoparticles using Capsicum annuum L. extract. Green Chemistry 9: $852-858$.

3. Dipanwita Maity Md, Masud Rahaman Mollick, Biplab Bhowmick, Dibyendu Mondal, Mrinal Kanti Bain, et al. (2012) Green Synthesis of Silver Nanoparticles Using Paederia foetida L. Leaf Extract and Assessment of their Antimicrobial Activities. International Journal of Green Nanotechnology 4: 230-239.
4. Gautam A, Mukherjee Shaibal, Ram S (2010) Controlled Novel Route to Synthesis and Characterization of Silver Nanorods. Journal of Nanoscience and Nanotechnology 10: 4329-4334. [crossref]

5. Sileikaite A, Prosycevas I, Puiso J, Juraitis A, Guobiene A (2006) Analysis of silver nanoparticles produced by chemical reduction of silver salt solution. Mater. Sci 12: 287-291.

6. Asta Sileikaite, Igoris Prosycevas, Judita Puiso, Asta Guobiene (2009) Analysis of Silver Nanoparticles Produced by Chemical Reduction of Silver Salt Solution. Materials Science 12.

7. Dubey SP, Lahtinen M, Sarkka H, Sillanpaa M (2010) Bioprospective of Sorbus aucuparia leaf extract in development of silver and gold nanocolloids. Colloid Surf. B 80: 26-33. [crossref]

8. Benjamin N. Philip, Andor J. Kiss and Richard E. Lee, Jr. (2011). The protective role of aquaporins in the freeze-tolerant insect Eurosta solidaginis: functional characterization and tissue abundance of EsAQP1. Journal of experimental biology 214: 848-857. [crossref]

9. Ravishankar Bhat, Sharanabasava Vishwanath Ganachari, Raghunandan Deshpande, Venkataraman Abbaraju (2013) Rapid Biosynthesis of Silver Nanoparticles Using Areca Nut (Areca catechu) Extract Under Microwave-Assistance. Journal of Cluster Science 24: 107-114.

10. Li G, He D, Qian Y, Guan B, Gao S, et al. (2012) Fungus-mediated green synthesis of silver nanoparticles using Aspergillus terreus. Int. J. Mol. Sci 13: 466-476. [crossref]

11. Shankar S, Rai A, Ahmad A, Sastry M (2004) Rapid synthesis of Au, Ag, and bimetallic Au core-Ag shell nanoparticles using Neem (Azadirachta indica) leaf broth. Journal of Colloid and Interface Science 275: 496-502. [crossref]

12. Kalpana D, Han JH, Park WS, Lee SM, Wahab R, et al. (2019) Green biosynthesis of silver nanoparticles using Torreya nucifera and their antimicrobial activity. Arab J Chem 12: 1722-1732.

\section{Citation:}

Sulochana MB, Parameshwar AB (2022) Biosynthesis of Silver Nanoparticles by Green Chemistry from Ectoine-compatible Solute. Mol Genet Res Open Volume 5(1): 1-6. 